\title{
Discovering obscure sightseeing spots by analysis of geo-tagged social images
}

\author{
$\operatorname{AUTHOR}(\mathrm{S})$ : \\ Zhuang, Chenyi; Ma, Qiang; Liang, Xuefeng; \\ Yoshikawa, Masatoshi
}

\section{CITATION:}

Zhuang, Chenyi ...[et al]. Discovering obscure sightseeing spots by analysis of geo-tagged social images. ASONAM '15: Proceedings of the 2015 IEEE/ACM International Conference on Advances in Social Networks Analysis and Mining 2015 2015: 590-595

ISSUE DATE:

2015-08-25

URL:

http://hdl.handle.net/2433/217604

\section{RIGHT:}

(c) ACM, 2015. This is the author's version of the work. It is posted here by permission of ACM for your personal use. Not for redistribution. The definitive version was published in "ASONAM '15, Pages 590-595",

http://dx.doi.org/10.1145/2808797.2809386.; この論文は出版社版でありません。引用の際には出版社版をご確認ご利 用ください。; This is not the published version. Please cite only the published version. 


\title{
Discovering Obscure Sightseeing Spots by Analysis of Geo-tagged Social Images
}

\author{
Chenyi Zhuang, Qiang Ma, Xuefeng Liang, Masatoshi Yoshikawa \\ Graduate School of Informatics, Kyoto University, \\ Yoshida-Honmachi, Sakyo-ku, Kyoto 606-8501, JAPAN \\ Email: zhuang@db.soc.i.kyoto-u.ac.jp, \{qiang, xliang, yoshikawa\}@i.kyoto-u.ac.jp
}

\begin{abstract}
In contrast to conventional studies of discovering hot spots, by analyzing geo-tagged images on Flickr, we introduce novel methods to discover obscure sightseeing spots that are less well-known while still worth visiting. To this end, we face two new challenges that the classical authority analysis based methods do not encounter: how to discover and rank spots on the basis of 1) popularity (obscurity level) and 2) scenery quality. For the first challenge, we estimate the obscurity level of a spot in accordance with the visiting asymmetry between photographers who are familiar with a target city and those who are not. For the second challenge, the behavior of both viewers who browsed the images and photographers are analyzed per each spot. We also develop an application system to help users to explore sightseeing spots with different geographical granularities. Experimental evaluations and analysis on a real dataset well demonstrate the effectiveness of the proposed methods.
\end{abstract}

\section{INTRODUCTION}

Benefiting from Social Networking Service (SNS) and advances in mobile devices, people can upload and share their experiences on the Internet. Geo-tagged images are one of the typical content generated by users. The vital information it contains provides researchers with excellent opportunities for discovering landmarks and moving patterns. For instance, GPS traces [1], images [2], check-ins [3], and tweets [4] are treated as different kinds of user votes to help gather tourism knowledge. Authority based analysis, like "rank-by-count" and "rank-by-frequency" in a vote manner, is the basis for most of these trip recommendation research.

More importantly, based on a survey by Zheng et al. [5], the growing geo-referenced and community-contributed media resources have generated huge amounts of detailed location and event tags, covering not only famous landmarks but also obscure locations. Because obscure locations always have not enough visits or votes on the Internet, the conventional authority based analysis, which is used to recommend popular locations, is not suitable.

By dividing sightseeing spots into four quadrants on the basis of their "popularity" and "sightseeing quality", we are trying to discover the obscure ones [6]. Located in the quadrant with high sightseeing quality but low popularity, an obscure sightseeing location is a considerable choice for in-depth travel to not only enjoy the beautiful scenery but also experience local culture, especially for the repeat tourists who have already visited the most famous places there.

In this paper, to a given scenery object, we propose a system to discover the obscure sightseeing locations with two novel
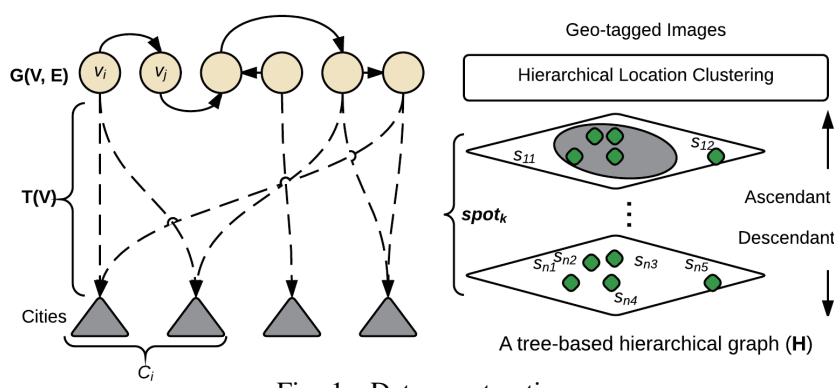

Fig. 1. Data construction

metrics of locations, "obscurity level" and "scenery quality", by analysis of geo-tagged images retrieved from Flickr [7]. In section III, we first devise novel methods to classify Flickr users by considering their familiarity with a given city. In contrast to conventional methods, we pay attention to users' properties and classify them into different groups to detect potential valuable spots. Then, we evaluate scenery quality of a spot by analyzing user behavior related to the images shot around it. The behavior of both viewers who browsed the social images (i.e., social appreciation) and photographers (i.e., photographers' attention) are considered in section IV. Implementations of the system are validated on the basis of field visits in Section V.

\section{OVERVIEW OF OUR SySTEM}

This section introduces our system, which is comprised of three components: data construction, obscure sightseeing spots mining, and recommendation.

\section{A. Data Construction}

Figure 1 illustrates the relationships between the main three data structures in our system.

Definition 1: A social network $G$ is a directed graph $G=$ $G(V, E)$, where $V$ is the photographer set of $v_{i}$ and $\mathrm{E}$ is the friendship set of $e\left(v_{i}, v_{j}\right)$ from $v_{i}$ to $v_{j}$.

Definition 2: A familiarity matrix $T_{i}$ is a mapping between a photographer $v_{i}$ and all the cities $C_{i}$ that s/he has been to. For example, $T_{i}[y=2014, c=$ kyoto $]=10, c \in C_{i}$ means that among $v_{i}$ 's images taken in Kyoto in 2014, there are 10 different dates detected from the images' taken dates.

Definition 3: A tree-based hierarchical graph $H$ is a collection of location clusters, denoted as $\operatorname{spot}_{k}$, with a geographical hierarchical structure. A $s_{\text {pot }}$ is a candidate sightseeing place, and all the images taken there are denoted as an image set $I_{k}$. 


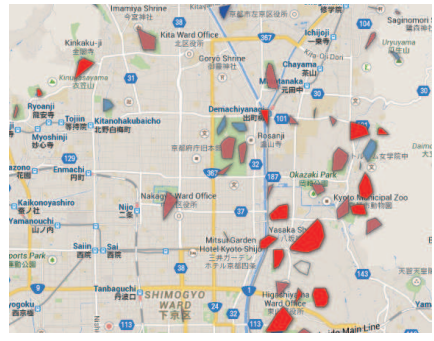

(a) Large Spots (zooming out)

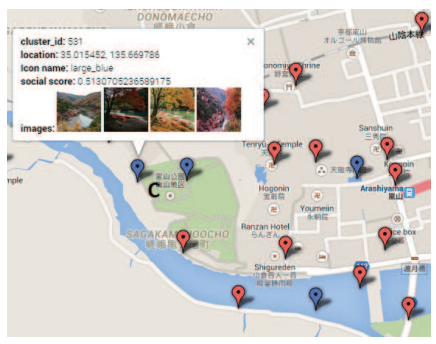

(b) Small Spots (zooming in)
Fig. 2. Exploring interfaces

\section{B. Obscure Sightseeing Spots Mining}

Obscurity Level Estimation: Given $G(V, E)$ and $T_{i}$, we first estimate each photographer $v_{i}$ 's familiarity with a particular city $c \in C_{i}$, based on which all the photographers are classified into a familiar group and an unfamiliar group. Then, on the basis of visiting frequency asymmetry between the photographers from these two groups, we estimate the obscurity level of each $\operatorname{spot}_{k}$.

Scenery Quality Evaluation: Two kinds of user behavior are identified to evaluate the scenery quality. Regarding the behavior of users who viewed target images, we evaluate a $\operatorname{spot}_{k}$ 's social appreciation to present how much on-line community members like some scenery around there. On the other hand, by analyzing the behavior of photographers who took target images, we try to evaluate the attention they have paid to some scenery at $\operatorname{spot}_{k}$.

\section{Recommendation}

Given a query, say "Kyoto, Maples", the recommendation component first labels all the related geo-tagged images (see Section IV-A). Then, as shown in Figure 2, a list of ranked sightseeing spots will be displayed on a Web map. By changing the zoom levels, a user can explore the spots in different geographical granularities. From deep blue (obscure) to deep red (popular), different colors indicate the obscurity levels of maple spots. By clicking any tags on the map, a user can view the images taken there, as well as the ranking information.

\section{OBSCURITY LEVEL ESTIMATION}

\section{A. Photographer Classification}

In this subtask, we will first introduce two complementary familiarity scores, $F_{s}$ and $F_{v}$, to classify photographers into the familiar group and unfamiliar group. Then, the relationship between these two scores will be discussed.

1) Social Network Based Familiarity Score: If a photographer is a resident, we assume s/he is familiar with the target city. However, few users specify their residency information on Flickr. Due to this, we propose a social network based method to calculate the familiarity of a photographer with a given city $c$. We assume that a photographer with many friends who are familiar with city $c$ will also be familiar with the city $c$.

$$
F_{s}(c)=\alpha_{B} \cdot G \cdot F_{s}(c)+\left(1-\alpha_{B}\right) d_{c} .
$$

By iterating the biased PageRank on the graph $G$, the familiarity value of a parent is split among its children, and the familiarity value of a child node is the sum of familiarity values propagated over its links. This is exactly consistent with our assumption. For the normalized bias distribution $d_{c}$, compared with the TruskRank [8], our system can automatically select good seeds due to whether a $v_{i}$ is a resident or not as detected from his/her profile. The entries of the vector $d_{c}$ that correspond to good seeds sum up to 1 . Given a city $c, F_{s}(c)$ is initialized to $d_{c}$ before the iteration of the above computation. $\alpha_{B}$ is the decay factor.

$\mathrm{Xu}$ et al. [9] have also proposed a similar assumption and proved that geographical nearby users are more probable to establish friendship relations using their dataset. However, the sensitivity (also known as recall) of this method would become low because of the exception that a potential good seed is pointed out by few good seeds. Thus, a complementary method is devised by considering photographers' visiting frequencies.

2) Visiting Frequency Based Familiarity Score: Since "familiarity" is time sensitive, on the basis of a $v_{i}$ 's familiarity matrix $T_{i}$ (see Definition 2) we calculate his/her "familiarity" of each $c \in C_{i}$ before a year $\lambda$. Three factors are considered:

1) For each year before $\lambda$, the proportion of days each $c$ accounts for is recorded in $T_{i}$;

2) Before $\lambda$, the reproducibility of cities is denoted as vector $\overrightarrow{\alpha_{i}}$. Assuming that before $\lambda$ we collect 10 years' worth of image data of $v_{i}$ in total, $\overrightarrow{\alpha_{i}}[c]=\frac{5}{10}$ if we detect that $v_{i}$ went to $c$ in five of the years;

3) The staleness of information. To identify whether $v_{i}$ has been to these cities recently, we introduce a diagonal matrix, $\omega_{i}(x, y) \forall x, y \in\{1,2, \ldots, n\}$, to characterize this feature. $n$ equals the number of detected years before $\lambda$.

Then, the score $F_{v}$ is computed as follows.

$$
F_{v}\left(v_{i}, c, \lambda\right)=\operatorname{diag}\left({\overrightarrow{\alpha_{i}}}^{T} \cdot\left(w_{i} \cdot T_{i}\right)\right)[c]
$$

where diag means matrix's diagonal vector, while $[c]$ is used to obtain the familiarity score of the target city $c$.

Obviously, the accuracy of $F_{v}$ highly depends on the scale of the familiarity matrix $T_{i}$. For a photographer who has uploaded few images, this method would not be effective. To cover as many situations as possible, we need to discuss the fusion of $F_{s}$ and $F_{v}$.

3) Score Integration: The most ideal situation is that $F_{v}$ can be propagated over the network $G(V, E)$. Namely, we can merge these two scores by using $F_{v}$ to select more good seeds (i.e., $d_{c}$ in Eq. 1) in the social network based method. However, for the cities (e.g., Kyoto) where locals rarely upload images to Flickr, exceptions are raised:

1) $F_{s}$ and $F_{v}$ are almost independent. That means a photographer with high visiting frequency is not necessarily a resident. Inversely, a resident is very likely to have a low visiting frequency based score.

2) In accordance with the first issue, there is no similarity of visiting frequency between $v_{i}$ and $v_{j}, \forall\left(v_{i}, v_{j}\right) \in E$. Namely, $F_{v}$ cannot be used to select more good seeds. 


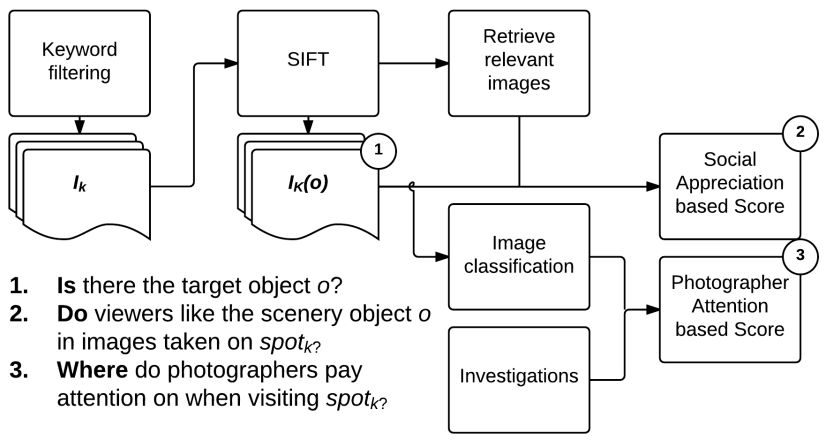

Fig. 3. Framework of ranking spots

For such exceptions that have no proportional relationships between $F_{s}$ and $F_{v}$, we find that the best way of combining the two scores in our experiments is to take the maximum:

$$
F\left(v_{i}, c, \lambda\right)=\max \left\{F_{s}\left(v_{i}, c\right), F_{v}\left(v_{i}, c, \lambda\right)\right\} .
$$

\section{B. Obscurity Score Calculation}

For a target city $c$, we first introduce a fuzzy set $\left\{U_{c}, m\left(v_{i}\right)=F\left(v_{i}, c, \lambda\right)\right\}$ to specify each $v_{i}$ 's familiarity on the basis of the integrated familiarity score $F\left(v_{i}, c, \lambda\right)$. Then, for a $\operatorname{spot}_{k}$, we define two vectors, $\phi_{f}^{s p o t_{k}}$ and $\phi_{\text {unf }}^{\text {spot }}$, to represent the percentage of both the familiar group and unfamiliar group who have visited $\operatorname{spot}_{k}$ in each year $y$ :

$$
\phi_{f}^{\text {spot }_{k}}[y]=\frac{w_{y}}{\left|U_{c}^{k}\right|} \sum_{v_{i} \in U_{c}^{k}} m\left(v_{i}\right) ; \phi_{\text {unf }^{\text {spot }}}^{\overrightarrow{p_{k}}}[y]=w_{y}-\phi_{f}^{\text {spot }_{k}}[y] .
$$

Finally, to calculate the time sensitive familiarity before year $\lambda$, the entries in $\phi_{f}^{s p o t} t_{k}$ and $\phi_{\text {unf }}^{s \overrightarrow{p o t} t_{k}}$ should include all the detected years before $\lambda$. We calculate spot $_{k}$ 's obscurity score by comparing the norms of these two vectors.

$$
\text { ObscurityScore }\left(\operatorname{spot}_{k}, \lambda\right)=\left\|\phi_{f}^{s \overrightarrow{p p t} t_{k}}\right\|-\left\|\phi_{\text {unf }^{\text {spot }}}^{\overrightarrow{p_{k}}}\right\| .
$$

\section{SCENERY Quality Evaluation}

Figure 3 presents the framework of evaluating a $\operatorname{spot}_{k}$ 's scenery quality. By observing the behavior of both viewers (i.e., who have viewed the images in $I_{k}$ ) and photographers (i.e., who have taken the images in $I_{k}$ ), we defined three progressive criteria. Along with this flow chart, the three criteria will be introduced in the remainder of this section.

\section{A. Image Labelling}

Given a query consisting a city $c$ and a scenery object $o$, the system labels the target images. Hereinafter, the set of all images about the scenery object $o$ that are taken at spot $_{k}$ is denoted as $I_{k}(o)$. Benefiting from Flickr's well implemented keyword based search function, the labelling has already obtained a good performance. For example, through manually statistics, the percentage of noise images in $I_{k}(o=$ cherryblossom) is about $7.72 \%$ on average.

We further applied SIFT [10] to reduce noise images in $I_{k}(o)$. In this model (see Figure 3), we use the key-points detected by SIFT to describe an image. For calculating the similarity, a fast nearest-neighbor algorithm, FLANN, is used to do the matching by considering both numbers of matches and match distance. When filtering, $I_{k}(o)$ itself is used as a set of references. After mutual match, the system removes the images obtaining the lowest similarity scores. By sampling in our ranking experiments, the percentage of noise images finally dropped to about $5.86 \%$.

After noise image filtering, we have met the first and most elementary criteria that there is the scenery object $o$ at $\operatorname{spot}_{k}$.

\section{B. Social Appreciation Based Analysis}

In this method, we use an intuitive value, the number of "Favs", to evaluate a scenery object's quality in a social appreciation manner. "Fave this photo" is a common user behavior on SNS websites including Flickr for friends and visitors to mark on a message or an image. In [11], Pedro et al. used the number of "Favs" as ground truth for building and testing their image attractiveness ranking models. However, our task is faced with two new problems: (1) few images may be taken at $\operatorname{spot}_{k}$; (2) a higher "Favs" may mean a beautiful image rather than beautiful scenery included in this image. In order to solve these two problems, two kinds of $I_{k}(o)$ 's related images are introduced.

Similar Content Images: Similar to a semi-supervised learning method having a small training set, we expand the existing data sets (i.e., $I_{k}(o)$ ) by detecting the similar content images. For each image in $I_{k}(o)$, we retrieve its most similar images by applying SIFT model as shown in Figure 3. The newly expanded image set is denoted as $I_{k}^{s}(o)$. High social appreciation on $I_{k}^{s}(o)$ anyhow shows that the $\operatorname{spot}_{k}$ is a good place for someone to take beautiful images about object $o$.

Context Images: We set the images from the same photosets to which an image $\eta \in I_{k}(o)$ belongs as its context images, denoted as $C_{\eta}$. Note that the image $\eta$ itself is not in $C_{\eta}$. In this sense, both $\eta$ and images in $C_{\eta}$ have a similar chance of being accessed by viewers. In addition, all these images are likely taken by the same photographer as well as similar shooting skills. Under these contexts, compared with $C_{\eta}$, higher social appreciation on $\eta$ suggests that $\eta$ contains beautiful scenery.

On the basis of these two related image sets, the final social appreciation based score consists of two parts. The first $I_{k}^{s}(o)$ based part is computed by the mean of ratios $\chi$ between an image $\eta$ 's number of "Favs" (i.e., vote $_{\eta}$ ) and view count (i.e., view $w_{\eta}$ :

$$
S_{1}\left(\operatorname{spot}_{k}, o\right)=\mu_{\chi}\left(I_{k}^{s}(o)\right) ; \quad \chi(\eta)=\frac{\text { vote }_{\eta}}{\text { view }_{\eta}} .
$$

The second $C_{\eta}$ based part is computed by $z$-scores:

$$
S_{2}\left(\operatorname{spot}_{k}, o\right)=\frac{1}{\left|I_{k}(o)\right|} \sum_{\eta \in I_{k}(o)} \frac{\chi(\eta)-\mu_{\chi}\left(C_{\eta}\right)}{\sigma_{\chi}\left(C_{\eta}\right)} .
$$

Function $\mu_{\chi}\left(C_{\eta}\right)$ and $\sigma_{\chi}\left(C_{\eta}\right)$ are used to respectively calculate the average $\chi$ and $\chi$ 's standard deviation on the image set $C_{\eta}$. Thus, positive and negative z-scores represent observation 
images above and below the mean, respectively. To combine $S_{1}$ and $S_{2}$, a weight factor $\omega_{c}$ is used to adjust discrimination.

$$
S A S\left(\operatorname{spot}_{k}, o\right)=S_{1}+\omega_{c} \cdot S_{2} .
$$

By proposing the $S A S$, we have met the second criteria of answering where to take beautiful pictures. By adapting this system to be a photography suggestion guide, the value of $\omega_{c}$ is somewhat a trade-off of recommending either attractive images or beautiful scenery. However, nobody can indeed know the reasons a viewer "Faves" an image seen when browsing the Web. Sometimes, we "Fave" images just because our friends have posted them. By proposing a heuristic method in the next section, we try to evaluate the scenery quality from a photographer's point of view.

\section{Photographer Attention Based Analysis}

For a scenery object $o$, it needs not only to be beautiful from a distance but also to have a delicate beauty up close. Figure 4(a.1) shows beautiful maple trees from far away, while in Figure 4(a.2) it shows a close-up of the beautiful leaves. The photographers' choices of taking distance view or closeup view images psychologically indicate their attention to a scenery object $o$.

Accordingly, we first propose a new method to divide scenery images into "distance view" and "close-up view", which exactly correspond to the two kinds of beauty above. Then, the regular patterns of photographers' attention when visiting the known sightseeing spots with beautiful scenery object $o$ are recognized. At last, we calculate the photographer attention based score, which indicates the degree of agreement with an observed regular pattern.

1) Image Classification: In the classification learning method, we use an image's edge histogram and HSV (hue, saturation, and value) shown in Figures $4 \mathrm{a}$ and $4 \mathrm{~b}$ as features to generate the training model. For the edge histogram, we find that a close-up view image has more clearer edges in the part where the photographer's camera focused. In other words, the background of a close-up view image is more blurred. In contrast, a distance view image has a gentle contrast throughout the whole image. For the HSV, we assume that the hue and saturation values of close-up view images of particular scenery are always located in a relatively fixed range (see Figure 4b). In contrast, there is no such rule for distance view images. This is due to that, for close-up view images of particular scenery, most content of the images is generally made up of the target scenery objects.

In accordance with our experiments described in Figure 4, our classification model reaches a stable accuracy that is close to $80 \%$. Torralba et al. [13] proposed a method to estimate the absolute depth of an image on the basis of the whole scene structure. For man-made scenes, the performance of depth estimations reaches $76 \%$, while the result is relatively lower on natural image sets (about $70 \%$ on average). When applied in our task of classifying scenery images into two categories: (1) "distance view"; or (2) "close-up view", our methods outperformed those of Torralba et al. [13].
2) Photographer Attention based Scenery Score: After the image classification, without loss of generality, we use the ratio of distance view images taken at a spot as $r_{o}$, which represents photographers' attention on the scenery object $o$ 's beauty from a distance.

Figure 5a shows two observations on the known spots having beautiful maples and cherry blossom. For maples, we find that photographers paid more attention to the up-close beauty. Conversely, cherry blossom's beauty from a distance attracted more attention compared with its up-close beauty.

Furthermore, by seeing the normal Q-Q plots in Figure $5 \mathrm{~b}$, we hypothesize that the two sample data sets come from the populations with normal distribution, since the points fall approximately along the 45-degree reference line. We performed the Lilliefors test, a kind of normality tests, to verify our hypothesis. By obtaining the $p-$ value $=0.26$ and 0.28 , which are higher than the significance value ( 0.05 or 0.1$)$, our hypothesis is statistically true.

As a result, based on the attention patterns recognized from spots having beautiful scenery $o$, a $\operatorname{spot}_{k}$ 's photographer attention based score is computed using a probability density function. For instance, since maples and cherry blossom both have the same pattern, we use the normal distribution functions to get their scores:

$$
P A S\left(\operatorname{spot}_{k}, o\right)=\frac{1}{\sigma_{o} \sqrt{2 \pi}} e^{-\frac{\left(r_{o}(k)-\mu_{o}\right)^{2}}{2 \sigma_{o}^{2}}} .
$$

$\mu_{o}$ and $\sigma_{o}$ are obtained by distribution fitting of investigation results on the target scenery object $o . r_{o}(k)$ is the ratio of distance view images taken at $s_{p o t}$. For different kinds of $o$, corresponding probability density functions are fitted. Intuitively, the $P A S$ indicates how well a $\operatorname{spot}_{k}$ can attract visitors' attention.

\section{EXPERIMENTS}

\section{A. Data Preparation}

From Flickr, we collected a total number of 929,403 images and the profiles of about 5,309,082 photographers. Using queries $q_{1}=$ "Kyoto, Maples" and $q_{2}=$ "Kyoto, CherryBlossom", two target sub-datasets are labelled by our system. For the corresponding datasets, they contain 6,950 maple images taken by 177 photographers and 4,576 cherry blossom images taken by 319 other photographers, respectively.

\section{B. Parameter Selection}

Spot clustering method: Both density based clustering algorithm (e.g., [14]) and agglomerative methods like K-means can be used for building the graph $H$ (see definition 3). Since the density based clustering would filter out a few sparsely distributed points that may be obscure, we utilized a centroid linkage based hierarchical clustering algorithm. We set the smallest cluster size to $2 \mathrm{~km}$ (i.e., leaves of $H$ ), which enables us to find some obscure sightseeing spots that exist in famous sightseeing areas. 


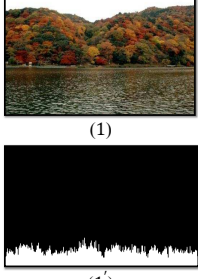

(1)

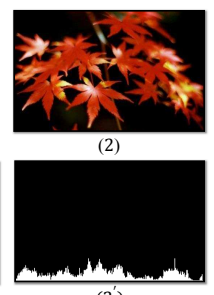

(2') (a) Image edge histogram $(\mathrm{EH})$

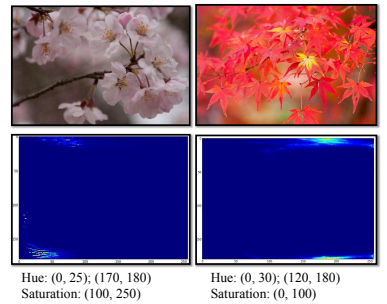

(b) Image HSV

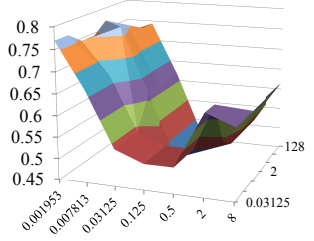

(c) EH\&HSV on Cherryset

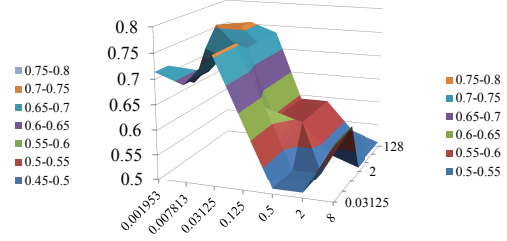

(d) EH\&HSV on Maple set

Fig. 4. Image classification. In (a), an image is vertically divided into 256 bins, and for each, the points of detected edges are counted to set up the histogram (i.e., EH). (b) presents the saturation (X-axis) and hue (Y-axis) distributions of close-up view images. Two original datasets, which repectively include 100 images, are labeled manually with either "distance view" or "close-up view". Then, 3-fold cross validation is utilized. (c) and (d) illustrate the effectiveness

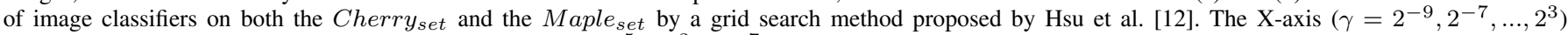
represents RBF kernel's parameter, while the Y-axis $\left(C=2^{-5}, 2^{-3}, \ldots, 2^{7}\right)$ stands for the penalty parameter in SVMs.

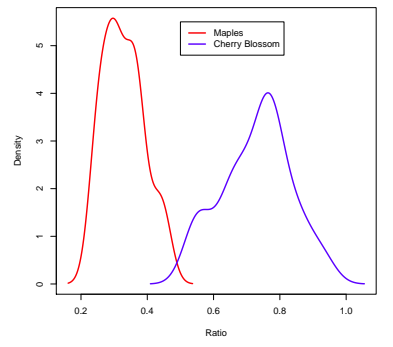

(a) Density of $r_{0}$

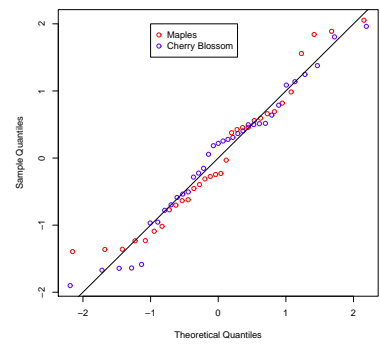

(b) Fitting verification of $r_{o}$

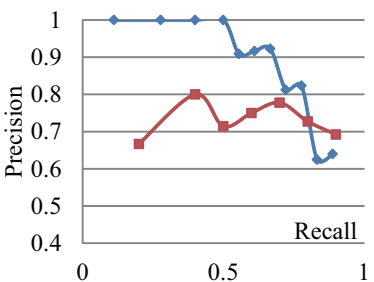

(a) "Kyoto, Maples"

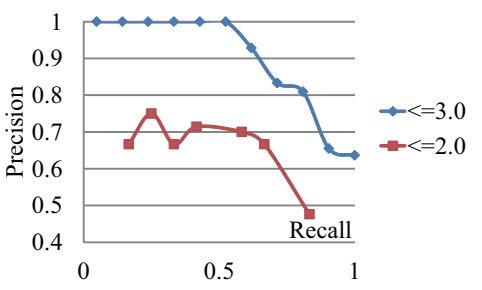

(b) "Kyoto, Cherry Blossoms"

Fig. 6. Precision-recall of discovering spots.

Fig. 5. Distribution fitting of sample data. Red: 22,421 geo-tagged maple set the threshold equal to 0.5 (i.e., $F_{s}\left(v_{i}, K y o t o\right) \geq 0.5$ ), the images taken at 32 spots with beautiful maples; Blue: 10,748 geo-tagged cherry blossom images taken at 35 spots with beautiful cherry blossom. TABLE I

DETAILS OF PHOTOGRAPHER CLASSIFICATION

\begin{tabular}{lc}
\hline \hline Target photographers No. & 177 \\
Known Kyoto locals No. & 18 \\
Recall on social network based method & $50 \%$ \\
Recall on visiting frequency based method & $77.8 \%$ \\
Recall on using both of the two methods & $83.3 \%$ \\
\hline \hline
\end{tabular}

$\boldsymbol{w}$ : Since both $w_{i}$ in Eq. 2 and $w_{y}$ in Eq. 4 have the same meaning of characterizing the staleness of information, on the basis of the actual effectiveness, we set them to the same exponential decay: $\frac{1}{2}^{(\lambda-y)}$. We set the $w_{c}$ in Eq. 8 to 1.0 , which means that the $S A S$ emphasizes more on the beauty of scenery rather than the attractiveness of images.

\section{Photographer Classification Evaluation}

Table I shows the detailed information. Among the target 177 photographers, we obtain 18 Kyoto locals according to their public profiles, while the other 159 photographers are unknown. For the social network based method (i.e., Eq. 1), we retrieve 26,567 their friends to establish the social network. For the visiting frequency based method (i.e., Eq. 2), we totally collected 395,053 images taken by them to calculate the familiarity matrices $T_{i}$. The "recall" in Table I means the fraction of familiar photographers that are discovered among the 18 known residents.

For nodes (photographers) in the social network, we detect 318 residents as good seeds based on their available profiles. Although there are few good seeds selected, "familiarity communities" are still detected among the network. When we

recall of this method reaches $50 \%$. For the second method, on the basis of the normalized $F_{v}\left(v_{i}, K\right.$ yoto, 2013$) \geq 0.5$, we obtain the recall of $77.8 \%$ among the 18 residents. The final recall obtained by considering both social network and visiting frequency (i.e., Eq. 3) is much higher, which reaches $83.3 \%$.

\section{Experiment on Obscurity Level Estimation}

In order to analyze the effectiveness of our method for estimating a spot's obscurity level (i.e., Eq. 5), we invited three residents who have lived in Kyoto for more than 20 years to label the discovered spots using an a five-point scale ranging from "0" for "not famous" to "4" for "very famous".

Figures $6 \mathrm{a}$ and $6 \mathrm{~b}$ illustrate precision-recall curves about the results of $q_{1}$ and $q_{2}$. The blue curve shows the experimental results when we regard the spots with the average score of the three respondents with a relevance score no more than 3.0, while the red one displays the corresponding results when the relevance score is no more than 2.0.

By getting leaves of the tree-based graph $H$, we obtain 582 maple and 368 cherry blossom spots. To reduce the workload of respondents, we choose 32 maple candidate spots and 33 cherry blossom candidate spots from which 17 and 18 discovered obscure ones are returned, respectively, to do the analysis. The blue curves in both figures indicate that the ObscurityScore can exclude almost all the "very famous spots". Furthermore, the red curves show that it still does well at discovering "not so famous" and "not famous" spots.

\section{E. Experiment on Ranking Obscure Spots}

Ground Truth: For $q_{1}$ and $q_{2}$, we obtained the ground truth through field visits conducted in November 2013 and April 
TABLE II

TWO CRITERIA OF SCENERY RANKING SCORES

\begin{tabular}{clc}
\hline \hline Criteria & Explanations & Ratings \\
$\mathrm{v}$ & Is the spot worth visiting or not. & $0,1,2,3$ \\
$\mathrm{r}$ & Is the spot worth recommending or not. & $0,1,2,3$ \\
\hline \hline
\end{tabular}
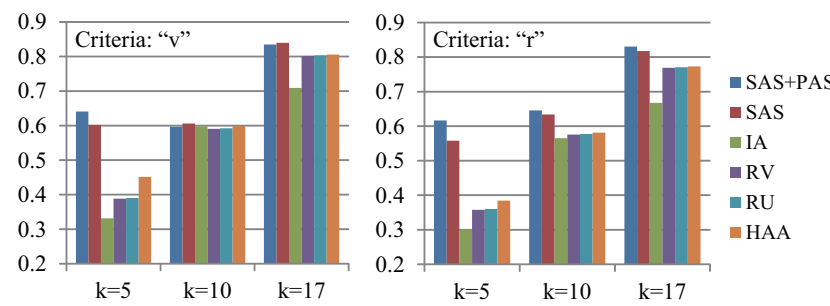

(a) NDCG on "Kyoto, Maples"
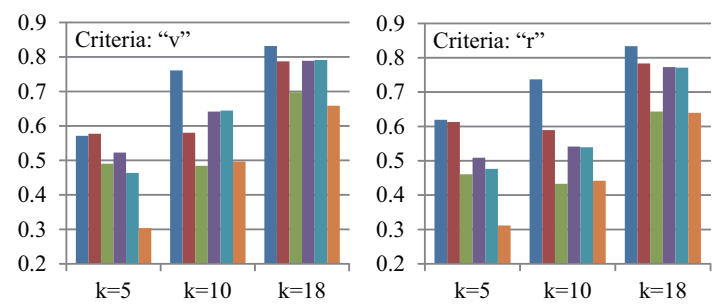

- SAS+PAS

- SAS

IA

$=\mathrm{RV}$

$=\mathrm{RU}$

(b) NDCG on "Kyoto, Cherry Blossom"

Fig. 7. NDCG values of the top- $k$ ranking results.

2014. For each of the selected 32 maple spots and 33 cherry blossom spots, we invited at least three subjects to perform field evaluation. Using Google Nexus 7, we asked them to record their visiting experiences on Twitter as well as take geotagged photos at each spot. Verified by these logs, we say that the human evaluation is credible. After that, they answered a questionnaire about their opinions of the spots they had visited. On the basis of the feedback, we defined two criteria to denote how well a discovered obscure spot meets tourist requirements shown in Table II.

We compare other 4 baseline methods with the $S A S$ (i.e., Eq. 8) and $P A S$ (i.e., Eq. 9):

- Image Attractiveness (IA): the image attractiveness ranking model proposed in [11];

- HITS based Authority Analysis (HAA): the user(hub)location(authority) graph based analysis in [1];

- Rank-by-Visits (RV): the accumulative counts of visits to the spot;

- Rank-by-Users (RU): the accumulative counts of unique users who have been to the spot.

By implementing these methods, we rank the $17+18$ obscure spots mentioned above.

NDCG based Evaluation: Figure $7 \mathrm{a}$ and $7 \mathrm{~b}$, respectively, depict the NDCG [15] of different measures on the two queries: $q_{1}$ and $q_{2}$. From the data shown in these figures, we can obtain the following results.

First, in contrast to the baseline methods, our proposed methods, $S A S+P A S$ and $S A S$, performed more effectively in the scenery quality based obscure spot ranking.
Second, the $P A S$ brings a significant improvement to the ranking only based on $S A S$. Since ranking obscure spots of having few images is very challenging, our experiments verified that the photographer attention based analysis could provide a stable effectiveness improvement.

Third, by comparing Figure $7 \mathrm{a}$ and $7 \mathrm{~b}$, the authority based analysis, $H A A$, fluctuated considerably. Namely, this series of conventional methods heavily depend on how much the corresponding raw data obtained.

Finally, the results related to $I A$ have verified our hypothesis that an attractive image doesn't mean beautiful scenery included in this image. As naive methods, $R V$ and $R U$ both performed better than expected, because our system has prefiltered out the noises (like crowded spots of having no target scenery) in the first step of ranking.

\section{CONCLUSION}

We proposed an obscure sightseeing spots discovery system based on two novel metrics, obscurity level and scenery quality. In the experiments, we employed human effort to obtain ground truth for the two query instances and further analyzed the performance of each proposed method by comparison.

\section{ACKNOWLEDGMENT}

This work is supported by JSPS KAKENHI Grant Numbers 25700033, 15J01402, and 15K00236.

\section{REFERENCES}

[1] Y. Zheng and X. Xie, "Learning travel recommendations from usergenerated gps traces," ACM TIST, vol. 2, no. 1, p. 2, 2011.

[2] W.-C. Chen, A. Battestini, N. Gelfand, and V. Setlur, "Visual summaries of popular landmarks from community photo collections," in ACM Multimedia, 2009, pp. 789-792.

[3] J. Liu, Z. Huang, L. Chen, H. T. Shen, and Z. Yan, "Discovering areas of interest with geo-tagged images and check-ins," in ACM Multimedia, 2012, pp. 589-598.

[4] K. Hasegawa, Q. Ma, and M. Yoshikawa, "Trip tweets search by considering spatio-temporal continuity of user behavior," in $D E X A$ (2), 2012, pp. 141-155.

[5] Y. Zheng, Z. Zha, and T. Chua, "Research and applications on georeferenced multimedia: a survey," Multimedia Tools Appl., vol. 51, no. 1, pp. 77-98, 2011.

[6] C. Zhuang, Q. Ma, X. Liang, and M. Yoshikawa, "Anaba: An obscure sightseeing spots discovering system," in ICME, 2014, pp. 1-6.

[7] Flickr apis. [Online]. Available: https://www.flickr.com/services/api/

[8] Z. Gyöngyi, H. Garcia-Molina, and J. O. Pedersen, "Combating web spam with trustrank," in $V L D B, 2004$, pp. 576-587.

[9] D. Xu, P. Cui, W. Zhu, and S. Yang, "Find you from your friends: Graph-based residence location prediction for users in social media," in ICME, 2014, pp. 1-6.

[10] D. G. Lowe, "Distinctive image features from scale-invariant keypoints," International Journal of Computer Vision, vol. 60, no. 2, pp. 91-110, 2004.

[11] J. S. Pedro and S. Siersdorfer, "Ranking and classifying attractiveness of photos in folksonomies," in $W W W, 2009$, pp. 771-780.

[12] C. wei Hsu, C. chung Chang, and C. jen Lin, "A practical guide to support vector classification," 2010.

[13] A. Torralba and A. Oliva, "Depth estimation from image structure," IEEE Trans. Pattern Anal. Mach. Intell., vol. 24, no. 9, pp. 1226-1238, 2002.

[14] M. Ester, H.-P. Kriegel, J. Sander, and X. Xu, "A density-based algorithm for discovering clusters in large spatial databases with noise," in $K D D$, 1996, pp. 226-231.

[15] K. Järvelin and J. Kekäläinen, "Cumulated gain-based evaluation of ir techniques,” ACM Trans. Inf. Syst., vol. 20, no. 4, pp. 422-446, 2002. 